\title{
Review Article \\ Resveratrol: Why Is It a Promising Therapy for Chronic Kidney Disease Patients?
}

\author{
Juliana F. Saldanha, ${ }^{1}$ Viviane de O. Leal, ${ }^{2}$ Peter Stenvinkel, ${ }^{3}$ José Carlos Carraro-Eduardo, ${ }^{4}$ \\ and Denise Mafra ${ }^{1}$ \\ ${ }^{1}$ Graduate Program in Medical Sciences, Federal Fluminense University (UFF), Niteroi, RJ, Brazil \\ ${ }^{2}$ Clinical Nutrition Department, State University of Rio de Janeiro (UERJ), Rio de Janeiro, RJ, Brazil \\ ${ }^{3}$ Department of Renal Medicine K56, Huddinge University Hospital, Karolinska Institutet, 14186 Stockholm, Sweden \\ ${ }^{4}$ University Hospital Antonio Pedro, Medicine Faculty of Federal Fluminense University (UFF), Niteroi, RJ, Brazil
}

Correspondence should be addressed to Juliana F. Saldanha; jusaldanha@hotmail.com

Received 13 June 2013; Revised 13 December 2013; Accepted 13 December 2013

Academic Editor: Ryuichi Morishita

Copyright (C) 2013 Juliana F. Saldanha et al. This is an open access article distributed under the Creative Commons Attribution License, which permits unrestricted use, distribution, and reproduction in any medium, provided the original work is properly cited.

Resveratrol, a phenolic compound found in various plants, including grapes, berries, and peanuts, shows promise for the treatment of cancer, aging, type 2 diabetes, and cardiovascular diseases. Resveratrol can promote transcription factor nuclear factor-erythroid 2related factor 2 (Nrf2) activation, increase the expression level of SIRT-1, which is a sirtuin family protein, and reduce mTOR pathway signaling. This compound has anti-inflammatory properties in that it inhibits or antagonizes the nuclear factor- $\kappa \mathrm{B}(\mathrm{NF}-\kappa \mathrm{B})$ activity, which is a redox-sensitive transcription factor that coordinates the inflammatory response. Inflammation and oxidative stress, which are common features in patients with chronic kidney disease (CKD), are interrelated and associated with cardiovascular disease and the progression of CKD itself. Because of the modulation of the mechanisms involved in the inflammatory-oxidative stress cycle, resveratrol could play an important role in controlling CKD-related metabolic derangements. Although resveratrol supplementation in theory is a promising therapy in this patient group, there are no studies evaluating its effects. Thus, the present review aims to describe the role of resveratrol in inflammation and oxidative stress modulation and its possible benefits to patients with CKD.

\section{Introduction}

Systemic inflammation and oxidative stress are nontraditional risk factors that are associated with premature cardiovascular disease commonly observed in patients with chronic kidney disease (CKD) [1,2]. Oxidative stress, which is characterized by an imbalance between oxidative free radical production and antioxidant capacity, appears to be the link between inflammation and cardiovascular disease in dialysis patients [3]. Moreover, in nondialysis CKD stage, oxidative mechanisms can be involved in renal tissue injury, in which the oxidative stress is associated with the progression of CKD itself $[4,5]$.

Resveratrol, a phenolic compound that is found in various plants, especially including red grapes and their derivatives [6], has demonstrated many beneficial effects, including anti-inflammatory and antioxidant roles by enhancing the production of antioxidant enzymes [7] and modulating nuclear factors involved in the inflammation-oxidative stress cycle $[8,9]$. However, no study has investigated the effects of resveratrol on patients with CKD. This review presents exciting evidence of the positive role of resveratrol in inflammation and oxidative stress control, and the study argues how resveratrol may represent an important link in the puzzle of CKD disorders.

\section{Inflammation and Oxidative Stress in CKD}

Inflammation and oxidative stress are interrelated conditions [10]. Oxidative free radicals are generated by phagocytic immune cells in response to inflammatory stimuli and released with proinflammatory cytokines, which in turn 


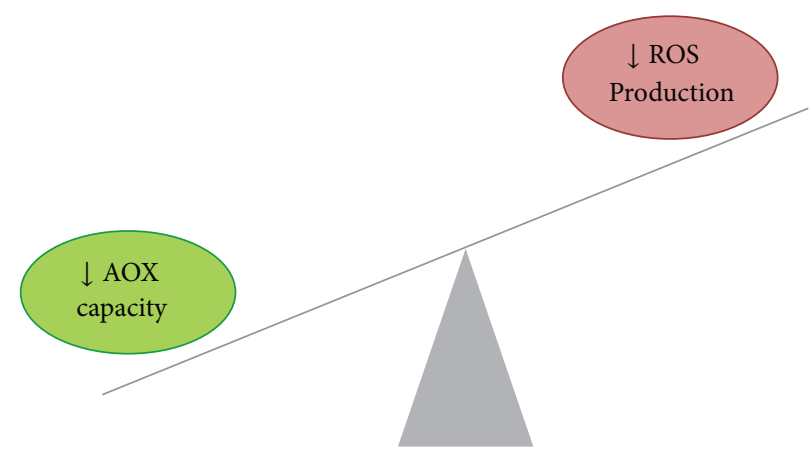

FIGURE 1: Oxidative stress: imbalance between antioxidant (AOX) capacity and reactive oxygen species (ROS) production.

amplify the generation of oxidants [11, 12]. To prevent the harmful effects of the oxidative status, enzymatic and nonenzymatic antioxidant systems counteract oxidative free radicals [11].

In $\mathrm{CKD}$, several features might contribute to a persistent state of inflammation and its prooxidant effects, such as proteinuria, reduced cytokine clearance, infections, comorbidities, underlying influences of clinical events, and dialysisrelated factors, including membrane bioincompatibility, dialysate backflow, and endotoxemia $[13,14]$. Patients with CKD demonstrate antioxidant defense deficiency because of the reduced consumption of vitamins and minerals containing antioxidants, such as vitamin $\mathrm{C}$ and selenium [15].

Oxidative stress results from this imbalance between oxidant generation and antioxidant defense mechanisms (Figure 1), leading to cell and tissue injury [16] and promoting the perpetuation of the inflammation-oxidative stress cycle by activating nuclear factor $\kappa \mathrm{B}(\mathrm{NF}-\kappa \mathrm{B})$, which is a redoxsensitive transcription factor that mediates the transcription of a large number of inflammatory genes coding for cytokines and adhesion molecules. Thus, when NF- $\kappa \mathrm{B}$ is activated, several cytokines are excessively produced, which leads to the formation of oxidative free radicals that establish a vicious cycle between inflammation and oxidative stress [17].

The chronic activation of $\mathrm{NF}-\kappa \mathrm{B}$ could predispose to atherosclerosis [18] because of its important role in inflammatory phenotypic changes in endothelial and smooth muscle cells [19]. In a recent study, Tilstra et al. [20] showed that NF$\kappa \mathrm{B}$ inhibition delays DNA damage-induced senescence and aging in mice. This transcription factor could be speculated to play a role in the human aging process [20]. Inflammation is a critical mechanism that promotes interlinked fibrosis and cellular injury in the renal interstitium [21], and, additionally, the decrease in renal function is accompanied by increased oxidative stress [22]. In this sense, chronic inflammation and oxidative stress features that are closely associated with $\mathrm{NF}-\kappa \mathrm{B}$ activation play a key role in the development and progression of CKD and its related disorders [23].

In contrast to NF- $\kappa \mathrm{B}$, transcription factor nuclear factorerythroid 2-related factor 2 ( Nrf2) is responsible for the constitutive and inducible expression of antioxidant response element-regulated genes [24] and is recognized to be a major cellular defense mechanism against oxidative stress [17].
When Nrf2 is released from its repressive cytosolic protein Keap 1, after it is translocated to the nucleus, Nrf2 activates genes that encode phase II detoxifying enzymes and antioxidant enzymes, such as glutathione peroxidase and heme oxygenase-1 $[17,25,26]$. In addition to reducing the expression of proinflammatory mediators, including cytokines and adhesion molecules, Nrf 2 appears to inhibit NF- $\kappa$ B activation [27] by regulating anti-inflammatory enzymes [17]. Thus, Nrf2 regulates cellular antioxidant responses and inhibits or antagonizes NF- $\kappa$ B actions $[17,24]$.

Different pharmacological and dietetic compounds are associated with Nrf2 activation. Among food compounds, rutin, quercetin [28], blackcurrant anthocyanins [29], and resveratrol [9] are demonstrated to promote Nrf2 activation.

\section{Resveratrol}

Resveratrol is a metabolite produced in more than 70 plant species in response to environmental stress [6], such as mechanical injury, microbial infection, and UV irradiation. Found in high concentrations in red grapes and their derivatives, resveratrol exists in nature in two isomeric forms (Figure 2): trans-resveratrol and cis-resveratrol [30]. Two phenol rings are linked by a styrene double bond to generate 3,5, $4^{\prime}$-trihydroxystilbene, which was first isolated in 1940 [31]. Although both isomers are biologically active, a majority of biological functions of resveratrol are attributable to transresveratrol, which is the more stable form. Initially, this compound attracted intense interest in 1992 when several cardioprotective effects were postulated to be associated with red wine [32] which implied that this benefit was an important factor in the French Paradox [33], that is, the observation that the French population has a low incidence of cardiovascular disease, despite having high saturated fat diet [34].

From that time, many biological effects have been assigned to resveratrol; the cardioprotective effects from resveratrol are the most known [35]. Resveratrol seems to improve vascular function by increasing nitric oxide synthesis and inhibiting its degradation [36], in addition to being able to increase the expression of antioxidant enzymes such as superoxide dismutase, catalase, and glutathione peroxidase [7]. Regarding the role of resveratrol in Nrf2 activation, 


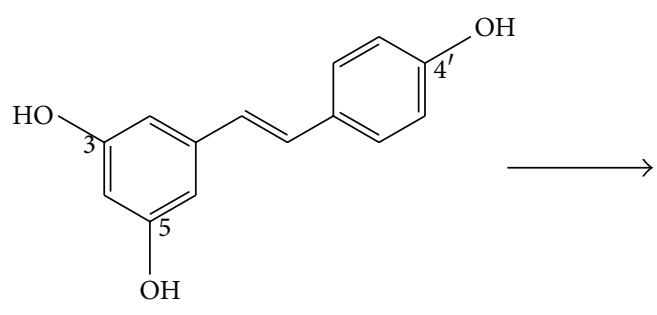

trans-Resveratrol

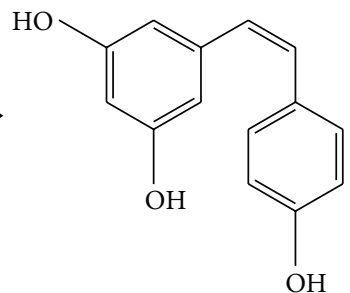

cis-Resveratrol

FIgURE 2: Chemical structure of resveratrol isoforms.

Ghanim et al. [9] evaluated in humans the effects of supplementation with a combination of resveratrol and grape skin polyphenols after a meal rich in carbohydrates and lipids. They observed an increase in Nrf2 and antioxidant enzyme expression, such as glutathione S-transferase [9]. In 2011, Palsamy and Subramanian [8] evaluated the renoprotective nature of resveratrol and observed that resveratrol administered orally to diabetic rats was capable of normalizing Nrf2 renal expression and related antioxidant factors, such as heme oxygenase-1 (HO-1) [8]. In this study, the resveratrol treatment was associated with the normalization of renal function and various inflammatory biomarkers, such as TNF and IL-6, in addition to being related to increased antioxidant activity.

In a randomized clinical trial, Timmers et al. [37] observed that resveratrol supplementation in obese subjects improved glucose tolerance and decreased hepatic steatosis and plasma inflammatory biomarkers [37]. In diabetic patients, resveratrol supplementation contributed to improving insulin sensibility, most likely by reducing oxidative stress [38].

Resveratrol seems to play a role in oxidative stress modulation by reducing NF- $\kappa$ B expression by sirtuins, which are proteins involved in the transcription, apoptosis, and energetic cell regulation [39-43]. The discovery of a homologous sirtuin (SIRT) family of proteins in the mammalian systems led to the realization that these molecules have beneficial effects in metabolism- and aging-related diseases. Sirtuins, $\mathrm{NAD}^{+}$-dependent deacetylases, are considered to be central modulators of longevity by playing an antioxidant role in preventing cardiovascular diseases. Until recently, researchers have identified seven homologous genes of the sirtuin family, SIRT-1 to SIRT-7; SIRT-1, with cellular nucleus localization, is the most studied $[44,45]$. SIRT-1 expression increases with caloric restriction, during fasting or food deprivation, or when cells are exposed to oxidative stress conditions and DNA damage [46, 47]; SIRT-1 expression seems to decrease under several inflammatory conditions by unknown mechanisms [48]. In humans, Cohen et al. [46] observed that SIRT-1 activation improves the apoptosis resistance of renal embryonic cells [46]; in vitro studies suggested that SIRT-1 has an important role in protecting renal medullar cells [44].

In an elegant study, Chen et al. [49] demonstrated that, in rats, resveratrol treatment ameliorated diabetic ketoacidosis and muscle protein degradation by the attenuation of elevated urinary methyl-histidine and plasma branched-chain amino acid levels [49]. In this study, the beneficial effects of resveratrol in diabetic rats were correlated with the activation of hepatic AMP-activated protein kinase and SIRT-1 expression, increases in hepatic and muscular mitochondrial biogenesis, and the inhibition of muscle NF- $\kappa \mathrm{B}$ activities. The authors concluded that resveratrol possesses multiple beneficial metabolic effects in insulin-deficient diabetic rats, particularly including effects involved in improving energy metabolism and reducing protein waste [49].

The mechanisms underlying the protective effects of resveratrol on various cardiovascular and metabolic disorders have not been established; however, evidence suggests that the inhibition of the mammalian target of rapamycin (mTOR) signaling pathway could play a role $[50,51]$. mTOR is a member of the PI 3-kinase-related protein kinase (PIKK) family that plays a critical role in the regulation of cell homeostasis in response to various upstream stimuli, such as growth factors, nutrients, and stress [52, 53].

Although several studies have suggested that activation of the SIRT-1 signaling pathway is essential for resveratrol action, Liu et al. [51] demonstrated that resveratrol inhibits insulin- and leucine-stimulated mTOR signaling in a SIRT1-independent manner [51]. The mTOR kinase nucleates two distinct protein complexes, termed mTORC1 and mTORC2. As presented in Figure 3, mTORC1 is stimulated by stress, oxygen, amino acids, energy, and growth factors that are acutely sensitive to rapamycin. mTORC1 promotes cell growth by inducing and inhibiting anabolic and catabolic processes, respectively, and drives cell-cycle progression and metabolism. mTORC2 is stimulated by growth factors and regulates cell survival, metabolism, and the cytoskeleton [54].

Several findings have indicated that resveratrol can negatively regulate mTOR activity via distinct mechanisms in response to different upstream stimulus [50]. Because mTOR activity is related to inflammatory and oxidative stress processes, its downregulation could attenuate these conditions.

\section{Resveratrol and CKD}

Given that inflammation and oxidative stress are implicated in the pathogenesis of cardiovascular disease in CKD and 


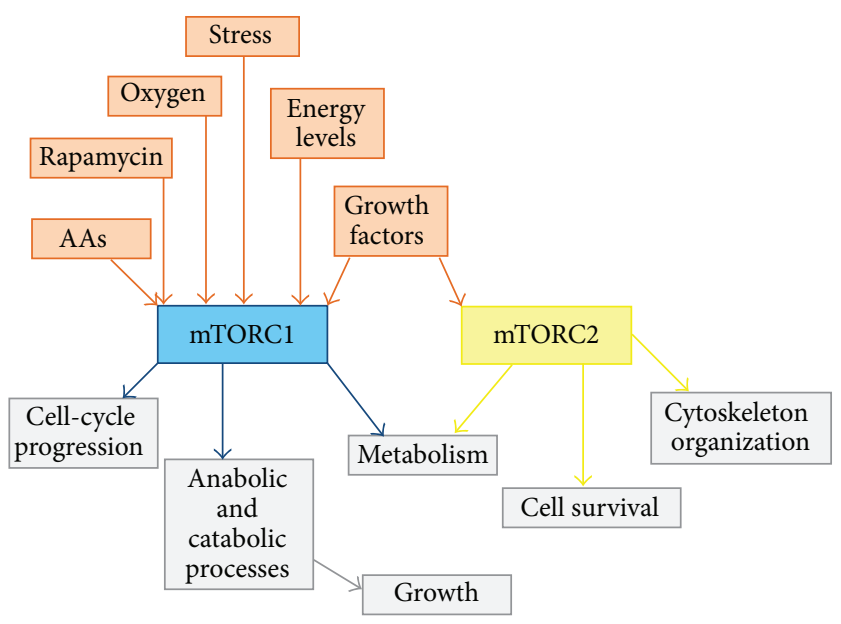

FIgURE 3: mTORC1 and mTORC2 complexes. AAs: amino acids.

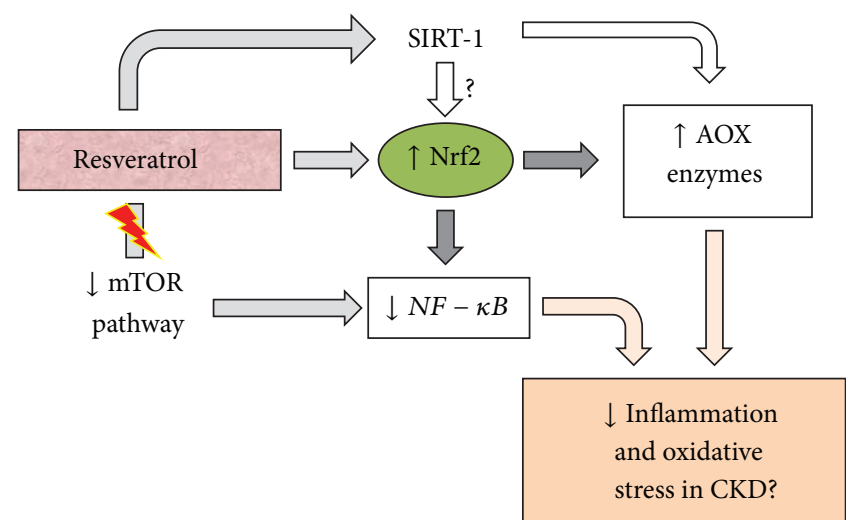

FIGURE 4: Scheme of resveratrol action mechanism to reduce inflammation and oxidative stress. AOX: antioxidant; CKD: chronic kidney disease.

other complications, compounds capable of attenuating these conditions, such as resveratrol, should attract particular interest in CKD treatment.

In mice, Liang et al. (2013) suggested that resveratrol treatment inhibits oxidative stress and renal interstitial fibrosis [55]. Additionally, clinical studies based on polyphenolcontaining food supplementation showed improvements in antioxidant activity and lipid profiles in hemodialysis patients $[56,57]$. However, until recently, no study has been developed to evaluate resveratrol effects in patients with $\mathrm{CKD}$, although it is plausible that resveratrol could provide several benefits to these patients by reducing inflammation and oxidative stress through SIRT-1 action, mTOR pathway inactivation, and Nrf2 and NF- $\kappa$ B factor modulation (Figure 4 ).

Although there are no reports of adverse effects related to the use of resveratrol in humans, even at high doses, clinical trials must be developed to explore resveratrol effects in CKD, considering its potential positive effects on systemic inflammation and oxidative stress control. Undeniably, resveratrol supplementation could represent a promising therapy to attenuate the progression of CKD, affect azotemia, and reduce morbidity and mortality by preventing or minimizing the risk of cardiovascular disease in patients with CKD.

\section{Conflict of Interests}

The authors declare that there is no conflict of interests.

\section{Acknowledgments}

The authors thank the National Council of Scientific and Technological Development (CNPq) and the Foundation of Research Support of Rio de Janeiro State (FAPERJ), E26/112.680/2012.

\section{References}

[1] S. Shastri and M. J. Sarnak, "Cardiovascular disease and CKD: core curriculum 2010," American Journal of Kidney Diseases, vol. 56, no. 2, pp. 399-417, 2010.

[2] P. Stenvinkel, "Inflammation as a target for improving health in chronic kidney disease," F1000 Medicine Reports, vol. 17, no. 1, Article ID M2-88, pp. 2-88, 2010.

[3] M. L. Wratten, D. Galaris, C. Tetta, and A. Sevanian, "Evolution of oxidative stress and inflammation during hemodialysis and their contribution to cardiovascular disease," Antioxidants and Redox Signaling, vol. 4, no. 6, pp. 935-944, 2002. 
[4] M. Kobayashi, H. Sugiyama, D.-H. Wang et al., "Catalase deficiency renders remnant kidneys more susceptible to oxidant tissue injury and renal fibrosis in mice," Kidney International, vol. 68, no. 3, pp. 1018-1031, 2005.

[5] G. Sener, K. Paskaloglu, H. Satiroglu, I. Alican, A. Kaçmaz, and A. Sakarcan, "l-Carnitine ameliorates oxidative damage due to chronic renal failure in rats," Journal of Cardiovascular Pharmacology, vol. 43, no. 5, pp. 698-705, 2004.

[6] G. J. Soleas, E. P. Diamandis, and D. M. Goldberg, "Resveratrol: a molecule whose time has come? And gone?" Clinical Biochemistry, vol. 30, no. 2, pp. 91-113, 1997.

[7] G. Spanier, H. Xu, N. Xia et al., "Resveratrol reduces endothelial oxidative stress by modulating the gene expression of superoxide dismutase 1 (SOD1), glutathione peroxidase 1 (GPx1) and NADPH oxidase subunit (Nox4)," Journal of Physiology and Pharmacology, vol. 60, pp. 111-116, 2009.

[8] P. Palsamy and S. Subramanian, "Resveratrol protects diabetic kidney by attenuating hyperglycemia-mediated oxidative stress and renal inflammatory cytokines via Nrf2-Keap1 signaling," Biochimica et Biophysica Acta, vol. 1812, no. 7, pp. 719-731, 2011.

[9] H. Ghanim, C. L. Sia, S. Abuaysheh et al., "An antiinflammatory and reactive oxygen species suppressive effects of an extract of Polygonum cuspidatum containing resveratrol," Journal of Clinical Endocrinology and Metabolism, vol. 95, no. 9, pp. E1-E8, 2010.

[10] J. Himmelfarb, P. Stenvinkel, T. A. Ikizler, and R. M. Hakim, "Perspectives in renal medicine: the elephant in uremia: oxidant stress as a unifying concept of cardiovascular disease in uremia," Kidney International, vol. 62, no. 5, pp. 1524-1538, 2002.

[11] F. Locatelli, B. Canaud, K.-U. Eckardt, P. Stenvinkel, C. Wanner, and C. Zoccali, "Oxidative stress in end-stage renal disease: an emerging treat to patient outcome," Nephrology Dialysis Transplantation, vol. 18, no. 7, pp. 1272-1280, 2003.

[12] B. Descamps-Latscha, T. Drüeke, and V. Witko-Sarsat, "Dialysis-induced oxidative stress: biological aspects, clinical consequences, and therapy," Seminars in Dialysis, vol. 14, no. 3, pp. 193-199, 2001.

[13] C. L. Meuwese, P. Stenvinkel, F. W. Dekker, and J. J. Carrero, "Monitoring of inflammation in patients on dialysis: forewarned is forearmed," Nature Reviews Nephrology, vol. 7, no. 3, pp. 166-176, 2011.

[14] J. J. Carrero and P. Stenvinkel, "Inflammation in end-stage renal disease-what have we learned in 10 years?" Seminars in Dialysis, vol. 23, no. 5, pp. 498-509, 2010.

[15] N. D. Vaziri, "Roles of oxidative stress and antioxidant therapy in chronic kidney disease and hypertension," Current Opinion in Nephrology and Hypertension, vol. 13, no. 1, pp. 93-99, 2004.

[16] A. Bonanni, I. Mannucci, D. Verzola et al., "Protein-energy wasting and mortality in chronic kidney disease," International Journal of Environmental Research and Public Health, vol. 8, no. 5, pp. 1631-1654, 2011.

[17] J. Kim, Y.-N. Cha, and Y.-J. Surh, "A protective role of nuclear factor-erythroid 2-related factor-2 (Nrf2) in inflammatory disorders," Mutation Research, vol. 690, no. 1-2, pp. 12-23, 2010.

[18] L. Hajra, A. I. Evans, M. Chen, S. J. Hyduk, T. Collins, and M. I. Cybulsky, "The NF- $\kappa$ B signal transduction pathway in aortic endothelial cells is primed for activation in regions predisposed to atherosclerotic lesion formation," Proceedings of the National Academy of Sciences of the United States of America, vol. 97, no. 16, pp. 9052-9057, 2000.
[19] A. Csiszar, M. Wang, E. G. Lakatta, and Z. Ungvari, "Inflammation and endothelial dysfunction during aging: Role of NF- $\kappa \mathrm{B}$," Journal of Applied Physiology, vol. 105, no. 4, pp. 1333-1341, 2008.

[20] J. S. Tilstra, A. R. Robinson, J. Wang et al. et al., "NF- $\kappa$ B inhibition delays DNA damade-induced senescence and aging in mice," The Journal of Clinical Investigation, vol. 122, no. 7, pp. 2601-2612, 2012.

[21] I. L. Noronha, C. K. Fujihara, and R. Zatz, "The inflammatory component in progressive renal disease-are interventions possible?" Nephrology Dialysis Transplantation, vol. 17, no. 3, pp. 363-368, 2002.

[22] A. Kuhad and K. Chopra, "Attenuation of diabetic nephropathy by tocotrienol: involvement of $\mathrm{NF} \kappa \mathrm{B}$ signaling pathway," Life Sciences, vol. 84, no. 9-10, pp. 296-301, 2009.

[23] I. Manabe, "Chronic inflammation links cardiovascular, metabolic and renal diseases," Circulation Journal, vol. 75, no. 12, pp. 2739-2748, 2011.

[24] L. Pedruzzi, M. B. Stockler-Pinto, M. Leite Jr., and D. Mafra, "Nrf2 keap-1 system versus NF- $\kappa$ B: the good and the evil in chronic kidney disease?" Biochimie, vol. 94, no. 12, pp. 24612466, 2012.

[25] K.-A. Jung and M.-K. Kwak, "The Nrf2 system as a potential target for the development of indirect antioxidants," Molecules, vol. 15, no. 10, pp. 7266-7291, 2010.

[26] S. Singh, S. Vrishni, B. K. Singh, I. Rahman, and P. Kakkar, "Nrf2-ARE stress response mechanism: a control point in oxidative stress-mediated dysfunctions and chronic inflammatory diseases," Free Radical Research, vol. 44, no. 11, pp. 1267$1288,2010$.

[27] H. Wang, L. Mao, L. Qiao, and X. Wang, "Disruption of Nrf2 enhances the upregulation of nuclear factor- $\kappa \mathrm{B}$ activity, tumor necrosis factor- $\alpha$, and matrix metalloproteinase- 9 after spinal cord injury in mice," Mediators of Inflammation, vol. 2010, Article ID 238321, 10 pages, 2010.

[28] R. Domitrovic, H. Jakovac, V. Vasiljev Marchesi et al., "Differential hepatoprotective mechanisms of rutin and quercetin in $\mathrm{CCI}_{4}$-intoxicated BALB/cN mice," Acta Pharmacologica Sinica, vol. 33, pp. 1260-1270, 2012.

[29] R. J. Thoppil, D. Bhatia, K. F. Barnes et al., "Black currant anthocyanins abrogate oxidative stress through Nrf2-mediated antioxidant mechanisms in a rat model of hepatocellular carcinoma," Current Cancer Drug Targets, vol. 12, no. 9, pp. 12441257, 2012.

[30] Y. Wu and F. Liu, "Targeting mTOR: evaluating the therapeutic potential of resveratrol for cancer treatment," Anti-Cancer Agents in Medicinal Chemistry, vol. 13, no. 7, pp. 1032-1038, 2012.

[31] M. J. Takaota, "The phenolic substances of white hellebore (Veratrum grandiflorum Loes. Fil.)," Journal of the Faculty of Science, Hokkaido Imperial University, vol. 3, pp. 1-16, 1940.

[32] E. H. Siemann and L. L. Creasy, "Concentration of the phytoalexin resveratrol in wine," American Journal of Enology and Viticulture, vol. 43, pp. 49-52, 1992.

[33] B.-L. Liu, X. Zhang, W. Zhang, and H.-N. Zhen, "New enlightenment of French paradox: resveratrol's potential for cancer chemoprevention and anti-cancer therapy," Cancer Biology and Therapy, vol. 6, no. 12, pp. 1833-1836, 2007.

[34] S. Timmers, J. Auwerx, and P. Schrauwen, "The journey of resveratrol from yeast to human," Aging, vol. 4, no. 3, pp. 146$158,2012$.

[35] B. Catalgol, S. Batirel, Y. Taga, and N. K. Ozer, "Resveratrol: french paradox revisited," Frontiers in Pharmacology, vol. 3, pp. $1-18,2012$. 
[36] H. Li and U. Fostermann, "Resveratrol: a multifunctional compound improving endothelial function," Cardiovascular Drugs and Therapy, vol. 23, pp. 425-429, 2009.

[37] S. Timmers, E. Konings, L. Bilet et al., "Calorie restrictionlike effects of 30 days of resveratrol supplementation on energy metabolism and metabolic profile in obese humans," Cell Metabolism, vol. 14, no. 5, pp. 612-622, 2011.

[38] P. Brasnyó, G. A. Molnár, M. Mohás et al., "Resveratrol improves insulin sensitivity, reduces oxidative stress and activates the Akt pathway in type 2 diabetic patients," British Journal of Nutrition, vol. 106, no. 3, pp. 383-389, 2011.

[39] X. Zhu, Q. Liu, M. Wang et al., "Activation of Sirt1 by resveratrol inhibits TNF- $\alpha$ induced inflammation in fibroblasts," PloS ONE, vol. 6, no. 11, p. e27081, 2011.

[40] H. Ghanim, C. L. Sia, K. Korzeniewski et al., "A resveratrol and polyphenol preparation suppresses oxidative and inflammatory stress response to a high-fat, high-carbohydrate meal," Journal of Clinical Endocrinology and Metabolism, vol. 96, no. 5, pp. 1409-1414, 2011.

[41] H. Yang, J. A. Baur, A. Chen, C. Miller, and D. A. Sinclair, "Design and synthesis of compounds that extend yeast replicative lifespan," Aging Cell, vol. 6, no. 1, pp. 35-43, 2007.

[42] S. M. Wyke, S. T. Russell, and M. J. Tisdale, "Induction of proteasome expression in skeletal muscle is attenuated by inhibitors of NF- $\kappa$ B activation," British Journal of Cancer, vol. 91, no. 9, pp. 1742-1750, 2004.

[43] K. T. Howitz, K. J. Bitterman, H. Y. Cohen et al., "Small molecule activators of sirtuins extend Saccharomyces cerevisiae lifespan," Nature, vol. 425, no. 6954, pp. 191-196, 2003.

[44] C.-M. Hao and V. H. Haase, "Sirtuins and their relevance to the kidney," Journal of the American Society of Nephrology, vol. 21, no. 10, pp. 1620-1627, 2010.

[45] E. Michishita, J. Y. Park, J. M. Burneskis, J. C. Barrett, and I. Horikawa, "Evolutionarily conserved and nonconserved cellular localizations and functions of human SIRT proteins," Molecular Biology of the Cell, vol. 16, no. 10, pp. 4623-4635, 2005.

[46] H. Y. Cohen, C. Miller, K. J. Bitterman et al., "Calorie restriction promotes mammalian cell survival by inducing the SIRT1 deacetylase," Science, vol. 305, no. 5682, pp. 390-392, 2004.

[47] J. T. Rodgers, C. Lerin, W. Haas, S. P. Gygi, B. M. Spiegelman, and P. Puigserver, "Nutrient control of glucose homeostasis through a complex of PGC- $1 \alpha$ and SIRT1," Nature, vol. 434, no. 7029, pp. 113-118, 2005.

[48] Y. Xiao, S. Karnati, G. Qian et al. et al., "Cre-mediated stress affects sirtuin expression levels, peroxisome biogenesis and metabolism, antioxidant and proinflamatory signaling pathways," PLoS ONE, vol. 7, no. 7, Article ID e41097, 2012.

[49] K. H. Chen, M. L. Cheng, Y. H. Jing, D. T. Y. Chiu, M. S. Shiao, and J. K. Chen, "Resveratrol ameliorates metabolic disorders and muscle wasting in streptozotocin-induced diabetic rats," American Journal of Physiology, vol. 301, pp. E853-E863, 2011.

[50] M. Liu and F. Liu, "Resveratrol inhibits mTOR signaling by targeting DEPTOR," Communicative and Integrative Biology, vol. 4, no. 4, pp. 382-384, 2011.

[51] M. Liu, S. A. Wilk, A. Wang et al., "Resveratrol inhibits mTOR signaling by promoting the interaction between mTOR and DEPTOR," The Journal of Biological Chemistry, vol. 285, no. 47, pp. 36387-36394, 2010.

[52] Q. Yang and K.-L. Guan, "Expanding mTOR signaling," Cell Research, vol. 17, no. 8, pp. 666-681, 2007.
[53] S. Wullschleger, R. Loewith, and M. N. Hall, "TOR signaling in growth and metabolism," Cell, vol. 124, no. 3, pp. 471-484, 2006.

[54] M. Laplante and D. M. Sabatini, "MTOR signaling in growth control and disease," Cell, vol. 149, no. 2, pp. 274-293, 2012.

[55] J. Liang, S. Tian, J. Han, and P. Xiong, "Resveratrol as a therapeuticagent for renal fibrosis induced by unilateral ureteral obstruction," Renal Failure. In press.

[56] P. Castilla, A. Dávalos, J. L. Teruel et al., "Comparative effects of dietary supplementation with red grape juice and vitamin $\mathrm{E}$ on production of superoxide by circulating neutrophil NADPH oxidase in hemodialysis patients," American Journal of Clinical Nutrition, vol. 87, no. 4, pp. 1053-1061, 2008.

[57] P. Castilla, R. Echarri, A. Dávalos et al., "Concentrated red grape juice exerts antioxidant, hypolipidemic, and antiinflammatory effects in both hemodialysis patients and healthy subjects," American Journal of Clinical Nutrition, vol. 84, no. 1, pp. 252$262,2006$. 


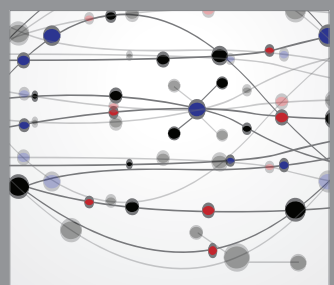

The Scientific World Journal
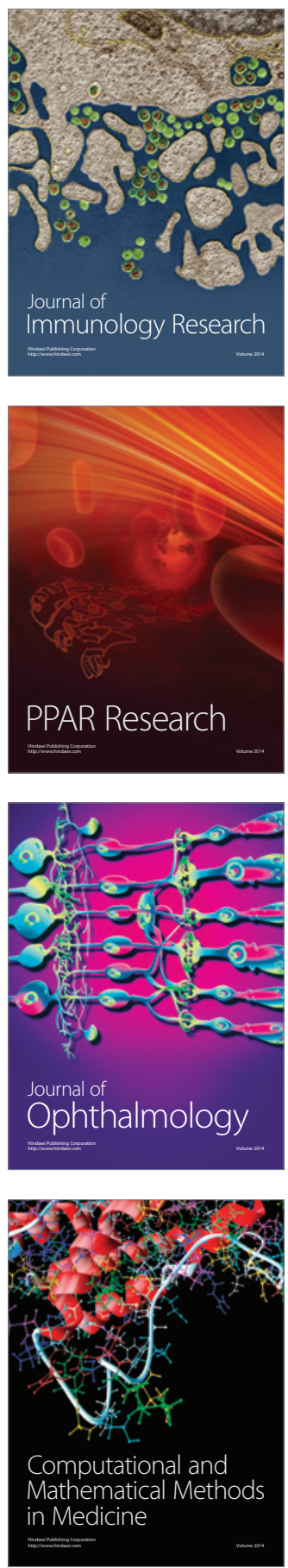

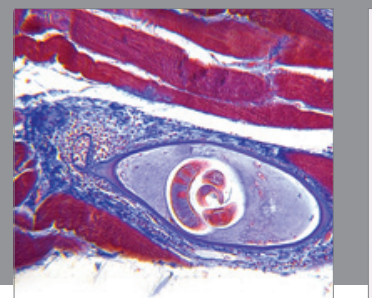

Gastroenterology

Research and Practice
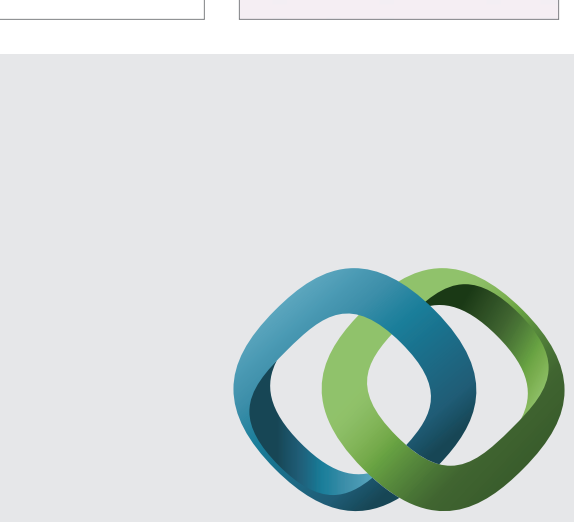

\section{Hindawi}

Submit your manuscripts at

http://www.hindawi.com
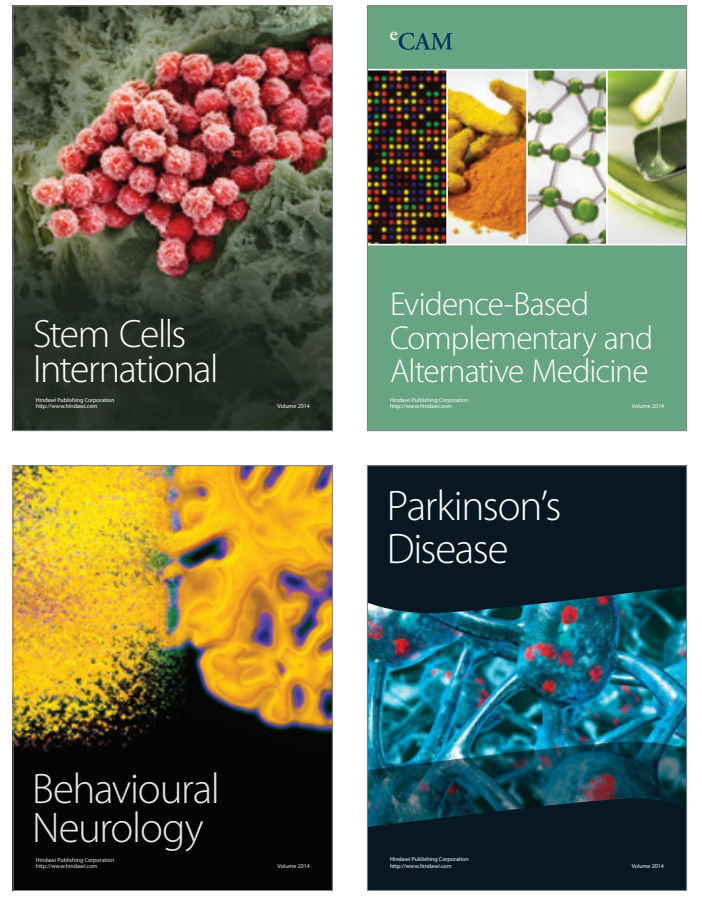
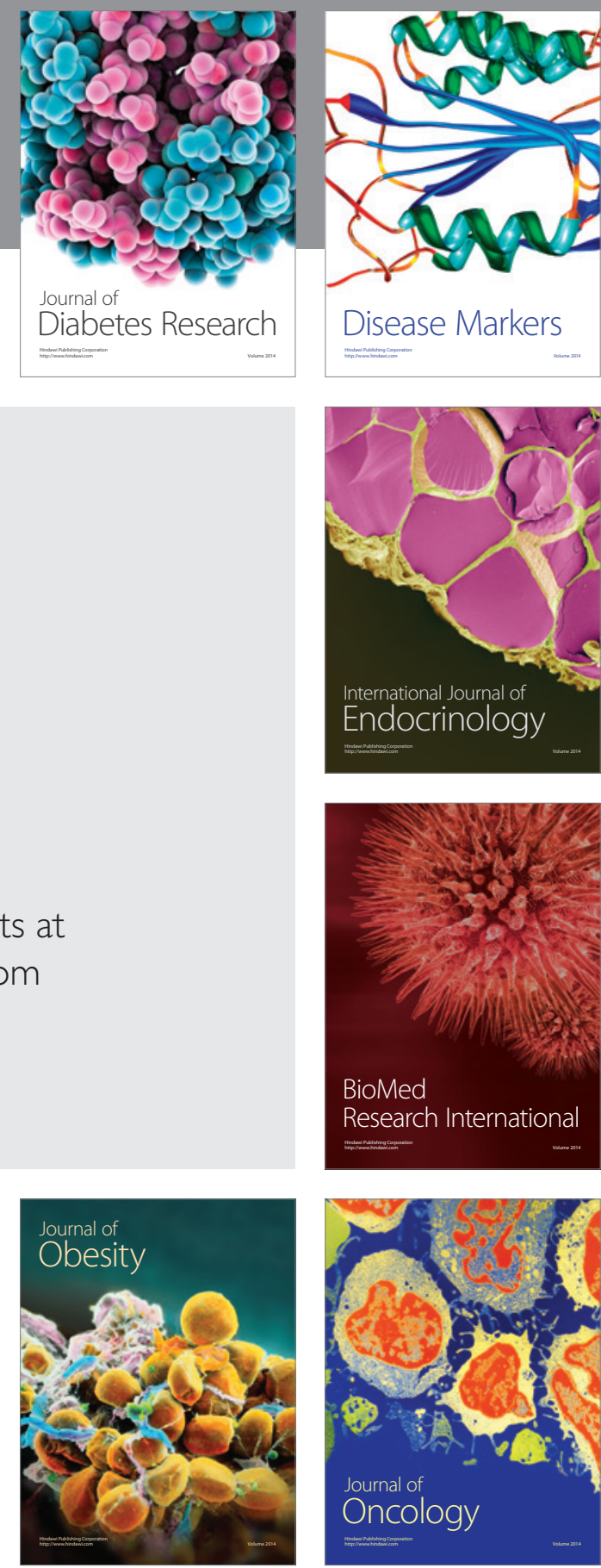

Disease Markers
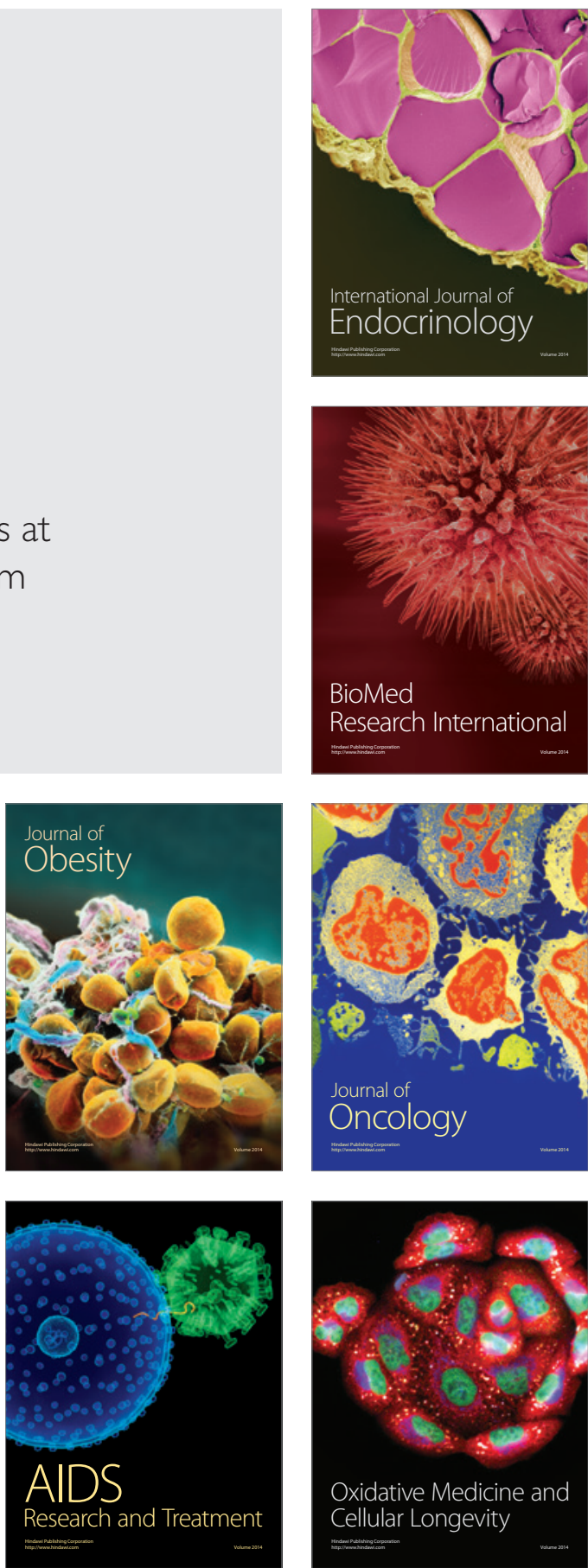